\title{
Die private Krankenversicherung - eine existenzielle Vorsorge
}

\author{
Um eine Krankenversicherung kommt in Deutschland keiner herum: Seit der Gesundheitsreform \\ 2007 muss sich jeder Bürger für den Fall einer Krankheit absichern. Nun stellt sich die Frage, ob \\ Krankheit gesetzlich oder privat versichert werden soll.
}

Während die Gesetzliche Krankenversicherung (GKV) - mit wenigen Ausnahmen - grundsätzlich allen offen steht, kann sich der Arbeitnehmer nur dann privat versichern, wenn sein Einkommen die sogenannte Jahresarbeitsentgeltgrenze übersteigt. Diese liegt derzeit bei 56.250 Euro. Selbstständige und Beamte sind davon ausgenommen, sie können in eine von ihnen favorisierte Private Versicherung (PKV).

Neben den Voraussetzungen unterscheiden sich die beiden Systeme auch in ihren Beiträgen. Bei der GKV ist der Beitrag vom Bruttoeinkommen abhängig. Wer mehr verdient, zahlt auch mehr. Der Beitrag in der PKV ist dagegen abhängig von Alter und Gesundheitszustand sowie dem gewünschten Versicherungsschutz.

\section{Vorteile der PKV}

Die PKV bietet eine ganze Reihe an Leistungen, die es in der GKV überhaupt nicht gibt: so zum Beispiel die freie Arztwahl inklusive Behandlung durch Privatärzte, eine Unterbringung im Einoder Zweibettzimmer, Chefarztbehandlung oder auch flexible Leistungen je nach Bedarf, um nur einige Privilegien zu nennen.

Auch wenn ein Wechsel in die PKV noch nicht möglich oder auch zurzeit nicht gewünscht ist, sollten die erheblichen Leistungslücken in der GKV unbedingt geschlossen werden. Vor allem bei der Absicherung von Verdienstausfall treten erhebliche Lücken auf.

Der Fall: Dr. Vogt, ein niedergelassener Zahnarzt, erlitt durch einen Fahrradsturz eine Rippenfraktur und eine Prellung am linken Daumen. Vom 3.7.2015 bis 18.9.2015 war er arbeitsunfähig; in der Zeit vom 26.8. bis 18.9. konnte er zu 50 Prozent arbeiten. Seine Konditionen bei der PKV waren ab dem 3. Tag 102,40 Euro, ab dem 8. Tag 204,80 Euro und ab dem 15. Tag 307,20 Euro. Soweit der Fall.

Was hat er durch die PKV beziehungsweise ohne deren Versicherungsschutz nicht erhalten?
Vom 3.7. bis 25.8 (54 Tage)

Vom 26.8. bis 18.9 (24 Tage)

Leistungen aus der GKV bei

100prozentiger Arbeitsunfähigkeit

Leistungen aus der GKV bei

50 prozentiger Arbeitsunfähigkeit

Einkommenslücke

16.765 Euro

Euro
GKV kann - gerade und vor allem bei jüngeren und im Praxisaufbau stehenden Zahnärzten - diese Einkommenslücke geschlossen werden.

\section{Optionstarif für Zahnmedizinstudenten}

Ein wichtiges Argument für eine private Krankenversicherung aus Sicht eines Existenzgründers ist auch die Flexibilität. $\mathrm{Zu} \mathrm{Be-}$ ginn einer Gründung heißt es unter betriebswirtschaftlichen Aspekten: auf die Kosten achten. Und da Zahnärzte in jungen Jahren bei der PKV in der Regel mit einem günstigen Tarif starten können, ist es möglich, im Vergleich zur GKV deutlich Kosten zu sparen. Zu einem späteren Zeitpunkt kann man dann den Tarif anpassen.

Apropos Kosten sparen: Bereits als Zahnmedizinstudent ist es überlegenswert, sich den späteren Wechsel in die PKV zu sichern. Diesen "Fahrschein in die Private" können Studenten mit einem sogenannten Optionstarif ziehen. Bei dessen Abschluss wird eine komplette Gesundheitsprüfung durchgeführt, und dieser Status ist festgeschrieben. Bei einem späteren Wechsel in die Vollversicherung erfolgt keine Gesundheitsprüfung. Zahnmedizinstudenten und Assistenzzahnärzte im FVDZ können diesen Optionstarif zu Sonderkonditionen erhalten. Alle Erkrankungen, die nach Abschluss des Optionstarifs auftreten, sind somit abgesichert. Einem Wechsel in eine private Vollversicherung, von der Basis- bis zur Top-Absicherung, steht keine Vorerkrankung im Weg.

Und noch etwas: Die Deutsche Ärzteversicherung ist als Standesversicherer ein profunder Kenner des Heilberufemarkts. Mit dem Freien Verband Deutscher Zahnärzte besteht seit Jahrzehnten ein Kooperationsabkommen, das den FVDZ-Mitgliedern eine Reihe exklusiver Vorteile bietet.

Sie wünschen weitere Informationen? Dann senden Sie uns den Servicecoupon auf DFZ-Seite 97 ausgefüllt zurück.

14.233,60 Euro 3.686,40 Euro

$1.155,--$ Euro

Fazit: Mit einer privaten Zusatzversicherung als Ergänzung zur

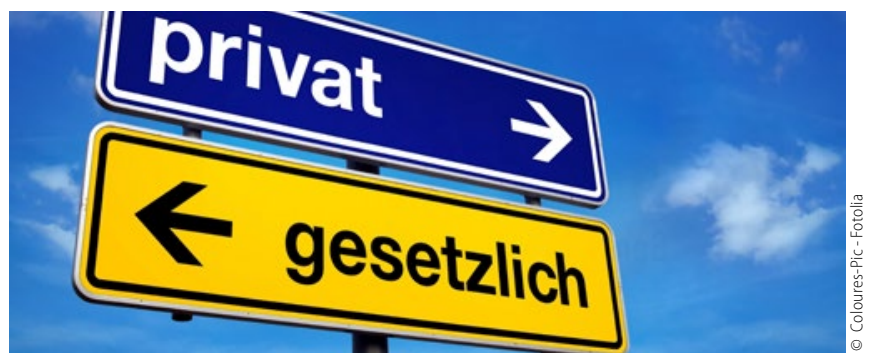

